\title{
Soldagem por Ponto no Estado Sólido de Ligas Leves
}

\author{
(Solid State Spot Welding of Lightweight Alloys)
}

\author{
Leonardo Contri Campanelli ${ }^{1}$, Nelson Guedes de Alcântara ${ }^{1}$, Jorge Fernandez dos Santos ${ }^{2}$ \\ ${ }^{1}$ Universidade Federal de São Carlos (UFSCar), Departamento de Engenharia de Materiais (DEMa), São Carlos, São Paulo, Brasil \\ -leoccampa@hotmail.com,nelsong@ufscar.br \\ ${ }^{2}$ Helmholtz-Zentrum Geesthacht GmbH, Instituto de Pesquisa em Materiais, Departamento de Processos de União no Estado Sólido \\ (WMP), Geesthacht, Alemanha-jorge.dos.santos@hzg.de
}

\begin{abstract}
Resumo
A recente preocupação quanto às mudanças climáticas vem impulsionando pesquisas em eficiência energética dos meios de transportes no sentido de reduzir a emissão de gases. Uma das principais soluções consiste na redução do peso estrutural através da aplicação de novos materiais, como as ligas leves de alumínio e magnésio. Entretanto, novos usos ficam muitas vezes limitados pela dificuldade de união desses materiais. A técnica de soldagem por fricção e mistura (FSW) é um processo de união no estado sólido que surge como uma alternativa viável para substituir ou complementar as tecnologias de união consagradas. Como uma junta contínua não é sempre a requisitada, duas tecnologias de união por ponto derivadas do FSW estão em desenvolvimento: soldagem por fricção e mistura por ponto (FSSW) e soldagem por fricção por ponto (FSpW). Além de fornecerem juntas de elevada resistência e praticamente isentas de defeitos, estas técnicas apresentam alta eficiência energética, curto ciclo de soldagem, facilidade de automação e compatibilidade com o meio-ambiente, fazendo frente às técnicas convencionais de união por ponto, como a soldagem por resistência por ponto (RSW) e a rebitagem.
\end{abstract}

Palavras-chave: Ligas leves; União no estado sólido; Soldagem por ponto.

Abstract: The recent concern about climate change has stimulated research into transport energy efficiency in order to reduce the emission of gases. One of the main solutions is to reduce the structural weight through the application of new materials, such as aluminum and magnesium lightweight alloys. However, new applications are often limited by the difficulty of joining these materials. Friction Stir Welding (FSW) is a solid state joining technique that emerges as a viable alternative to replace or complement the established joining technologies. As a continuous weld is not always requested, two spot welding technologies derived from FSW are under development: Friction Stir Spot Welding (FSSW) and Friction Spot Welding (FSpW). Besides providing almost defect-free and high strength joints, these techniques exhibit high energy efficiency, short welding cycle, ease of automation and environmental compatibility, competing against the conventional spot joining techniques, such as Resistance Spot Welding (RSW) and riveting.

Keywords: Lightweight alloys; Solid state joining; Spot welding.

\section{Introdução}

A preocupação mundial referente às questões ambientais tem conduzido à busca por soluções que permitam reduzir o consumo de combustíveis e a consequente emissão de gases poluentes pelos meios de transporte. Algumas possibilidades incluem a utilização de fontes renováveis de energia, melhorias na eficiência dos motores e na aerodinâmica e redução de peso, sendo esta última uma excelente opção de custo-benefício [1]. A redução do peso das estruturas está diretamente relacionada à utilização de materiais leves que apresentem uma alta resistência específica. As ligas de alumínio (Al) e de magnésio ( $\mathrm{Mg}$ ) se apresentam como opções naturais em função dos baixos valores de densidade, além de apresentarem excelente usinabilidade e

(Recebido em 01/08/2011; Texto final em 08/08/2011) possibilidade de reciclagem.

Os atributos das ligas de $\mathrm{Al}$ e $\mathrm{Mg}$ são de grande interesse das indústrias automobilística e aeronáutica, porém o emprego de tais materiais torna-se muitas vezes inviável em função da dificuldade de união. Algumas características metalúrgicas intrínsecas dificultam a obtenção de uma junta de alta qualidade por meio de processos convencionais de soldagem. Dado o potencial de aplicação, novas alternativas de processamento vem sendo estudadas nas últimas décadas. Desenvolvida em 1991 pelo The Welding Institute (TWI) na Inglaterra [2], a tecnologia de soldagem por fricção e mistura (Friction Stir Welding, FSW) tem sido aplicada com sucesso na união de diversas ligas metálicas, incluindo metais dissimilares. O processo, realizado no estado sólido, envolve essencialmente calor por fricção e deformação plástica severa, decorrentes da interação entre uma ferramenta não-consumível e a superfície da peça a ser soldada [3].

O fato de ser um processo no estado sólido elimina uma série de limitações associadas aos processos que envolvem a fusão 
do metal de base, tais como trincamento a quente, porosidade, respingos, entre outros [4]. Diversos autores referem-se ao FSW como uma tecnologia "verde" em função do menor consumo de energia e da compatibilidade com o meio-ambiente, não havendo a utilização de insumos, como gases de proteção e fluxo, ou de material de adição, reduzindo inclusive o custo de processo. $\mathrm{O}$ maior sucesso do FSW é atribuído à possibilidade de união de materiais anteriormente considerados não-soldáveis, tais como as ligas de Al das séries $2 \mathrm{XXX}$ e $7 \mathrm{XXX}[3,5]$. Embora o FSW tenha se tornado rapidamente uma tecnologia industrial importante, uma solda contínua não é sempre necessária para satisfazer os requisitos de desempenho de um produto.

Duas tecnologias derivadas do FSW, e que, portanto, reúnem todas as vantagens em relação aos processos convencionais, foram recentemente desenvolvidas para permitir a união por ponto de ligas metálicas: soldagem por fricção e mistura por ponto (Friction Stir Spot Welding, FSSW) e soldagem por fricção por ponto (Friction Spot Welding, FSpW). Na atualidade os setores automobilístico e aeronáutico empregam na união por ponto as técnicas de soldagem por resistência por ponto (Resistance Spot Welding, RSW) e de rebitagem, as quais acarretam, entre outros, um custo operacional elevado (alto consumo de energia) e o aumento do peso das estruturas, respectivamente [6]. O propósito deste trabalho é apresentar as características fundamentais dos processos FSSW e FSpW, aplicados principalmente em ligas metálicas leves.

\section{Soldagem por Fricção e Mistura por Ponto}

A soldagem por fricção e mistura por ponto (Friction Stir Spot Welding, FSSW) é um processo de união no estado sólido derivado do FSW com a particularidade de não haver o movimento transversal da ferramenta. Desenvolvido em 2001 pela empresa Mazda Motor Corporation no Japão [7], o processo é realizado em quatro etapas distintas por uma ferramenta cilíndrica nãoconsumível constituída por uma camisa (em inglês, shoulder) e um pino central sobressalente. A Figura 1 apresenta um esquema das quatro etapas do processo.

Inicialmente a ferramenta rotaciona até atingir uma velocidade desejada, seguindo-se a aplicação de uma força axial para proporcionar a penetração do pino na parte superior das chapas a serem soldadas dispostas em configuração de sobreposição. $\mathrm{O}$ calor gerado pelo atrito entre a ferramenta e a chapa promove a plastificação e o amaciamento do material,

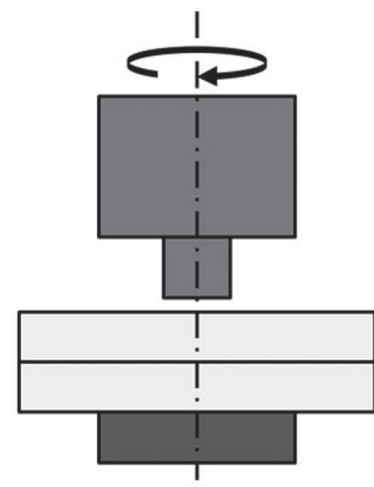

(a)

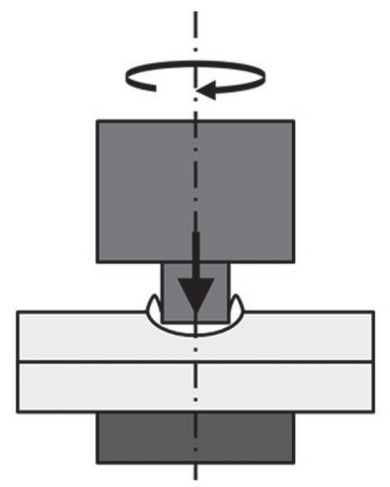

(b)

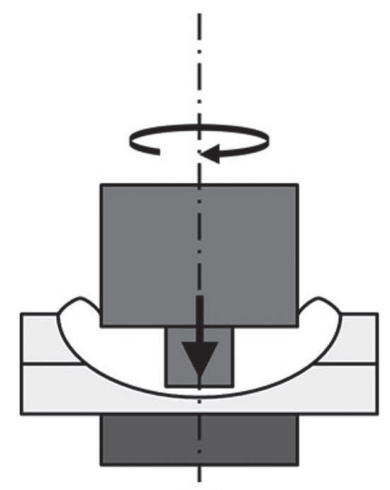

(c) facilitando a penetração subsequente, que procede até que a profundidade pré-determinada seja atingida. Após a penetração completa do pino, o contato entre a camisa e a superfície da chapa, além de resultar em um aporte térmico mais acentuado, origina uma força de compressão que consolida a ligação metalúrgica ao redor do pino. A mistura do material é otimizada durante um tempo de operação (em inglês, $d$ well time) no qual a ferramenta é mantida exclusivamente em rotação. O processo é finalizado com a remoção da ferramenta, obtendo-se uma solda com um furo característico.

O fluxo do material plastificado durante o processo FSSW [811] inicia-se quando o pino força-o para baixo, fazendo com que o material da chapa inferior se desloque para cima em direção à chapa superior. Com o contato entre a camisa e a chapa, conforme mostrado esquematicamente na Figura 2, o material localizado abaixo da camisa se move para a superfície do pino (movimento 1), deslocando-se de modo adjacente à superfície (movimento 2) como resultado da força de arraste decorrente da rotação dos filetes (pino com perfil de rosca). A incorporação de material de ambas as chapas, superior e inferior, se processa na superfície do pino antes que a mistura de materiais se desloque para baixo por meio dos filetes de rosca. Ao atingir a extremidade do pino, o material é lançado para fora e forçado para cima, retornando em direção ao pino por meio de um movimento espiral (movimento 3). O mecanismo de transporte descrito repete-se para distâncias maiores da periferia do pino para o desenvolvimento de uma zona de mistura. Para uma ferramenta com perfil liso, a ausência dos filetes de rosca no pino restringe consideravelmente o fluxo de material na direção vertical.

Assim como o processo FSW, a microestrutura de ligas leves soldadas por FSSW pode ser dividida em três regiões distintas além do metal de base (MB): zona recristalizada dinamicamente ou zona de mistura $(\mathrm{ZM})$, zona termo-mecanicamente afetada (ZTMA) e zona termicamente afetada (ZTA) $[12,13]$. A Figura 3 apresenta uma junta típica obtida por FSSW. A delimitação entre as diferentes zonas de solda é apenas uma aproximação. A extensão destas regiões depende não somente do material que está sendo soldado, mas também da combinação dos diferentes parâmetros de processo. Uma grande evolução microestrutural é dada pelo fato da ZM praticamente não apresentar defeitos metalúrgicos, ao contrário do processo RSW que resulta em uma quantidade de poros bastante significativa [12].

A região mais distante do cordão de solda não é afetada pelo calor e pela taxa de deformação gerada no processo, de forma

Figura 1 - Ilustração esquemática das quatro etapas do FSSW: (a) rotação, (b) penetração, (c) mistura e (d) remoção da ferramenta. 

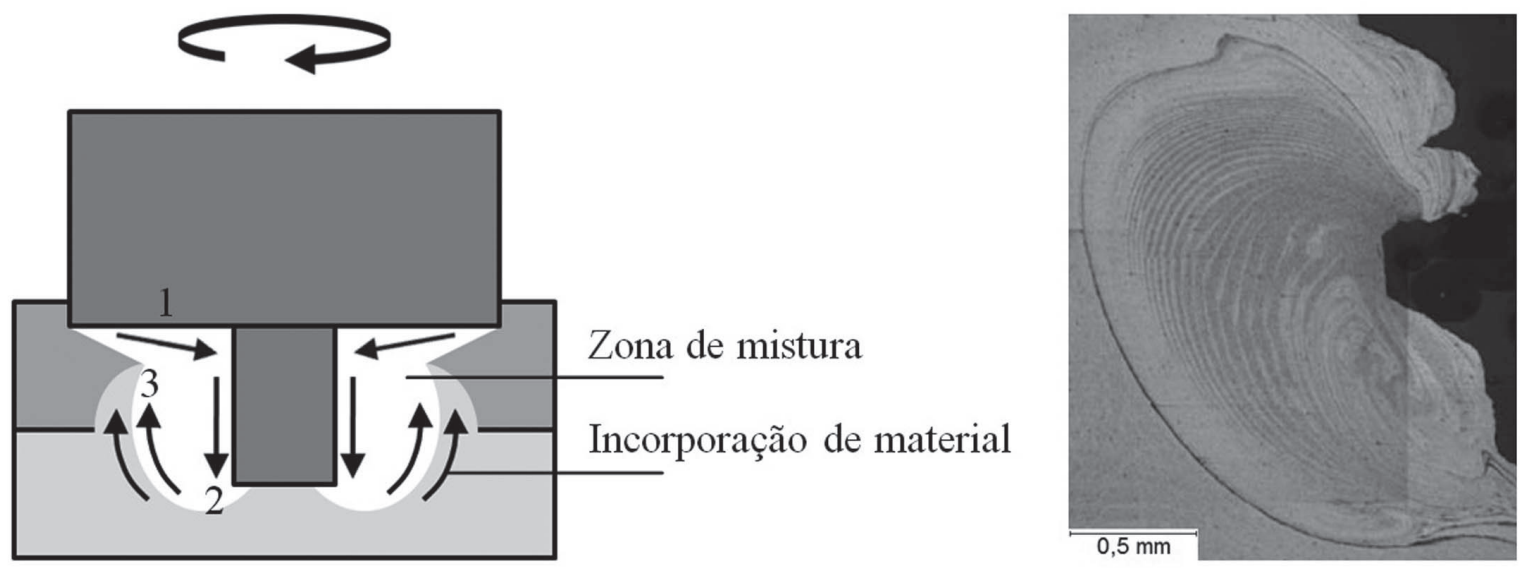

Figura 2 - Esquema do modelo de fluxo de material durante o FSSW, com destaque à direita para a zona de mistura adjacente ao pino [11].
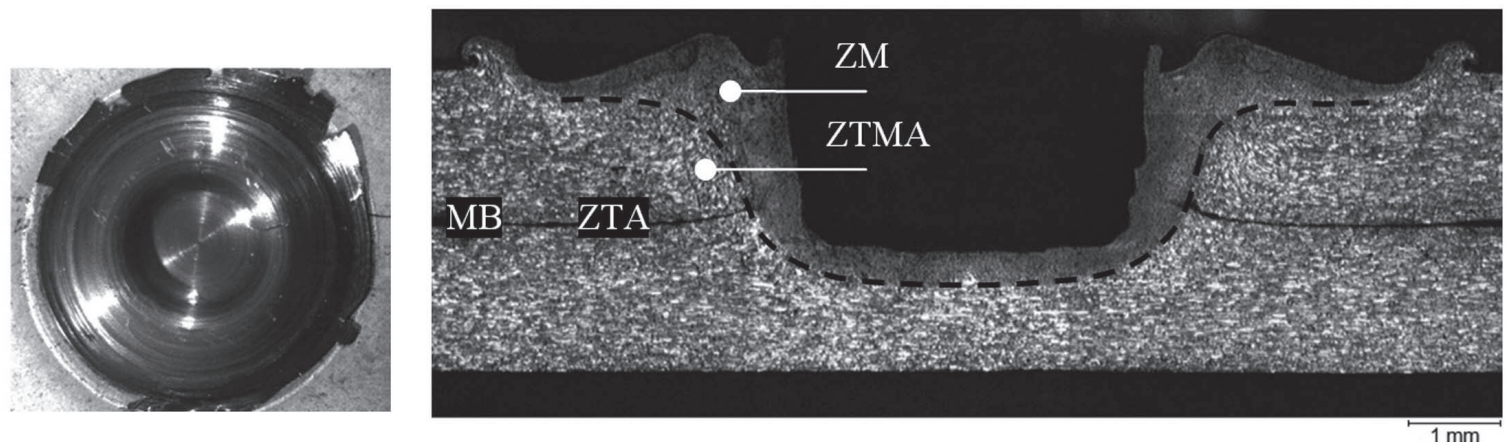

Figura 3 - Aspecto visual e macrografia da seção transversal de uma junta de Al 7075-T6 soldada por FSSW [14].

que as características dos grãos do MB devem-se exclusivamente ao processamento e/ou tratamento térmico anterior ao processo. Embora apresente uma diferença muito pequena em relação ao MB, a ZTA pode apresentar crescimento de grão ou então alguma transformação metalúrgica decorrente unicamente do aporte térmico a que a microestrutura é submetida, como a alteração na estrutura dos precipitados. $\mathrm{Na}$ ZTMA, região de transição entre o metal original e a região recristalizada, a deformação plástica, embora em grau insuficiente para que a recristalização ocorra, gera um padrão de fluxo ascendente da microestrutura. Em geral, ocorre o processo de recuperação e, eventualmente, a desestabilização do tratamento térmico da liga. A ZM experimenta um grau severo de deformação plástica e de aquecimento, suficientes para que a recristalização dinâmica se processe, resultando em uma microestrutura composta por pequenos grãos recristalizados e equiaxiais. A geometria da ZM varia com o perfil da ferramenta e os parâmetros de soldagem.

A primeira aplicação industrial do FSSW pela Mazda ocorreu em 2003 na montagem das portas traseiras e do capô em Al, em substituição ao RSW, do veículo modelo RX-8. Em 2005, o processo passou a ser empregado também na tampa do portamalas do modelo MX-5 [15]. A Figura 4 mostra alguns exemplos de aplicação. Embora o tempo de ciclo seja aproximadamente o mesmo para ambas as tecnologias, o FSSW proporciona uma redução drástica no consumo de energia, a qual é necessária somente para a rotação da ferramenta e aplicação da força axial, e requer um baixo investimento em maquinário. $\mathrm{O}$ processo elimina o ar comprimido e as correntes elétricas intensas essenciais para o RSW, reduzindo o consumo de energia em
$99 \%$ na união de ligas de $\mathrm{Al}$ e em $80 \%$ na união dos aços. A simplicidade dos equipamentos representa uma redução de $40 \%$ nos investimentos em comparação ao RSW [16,17].

\section{Soldagem por Fricção por Ponto}

A soldagem por fricção por ponto (Friction Spot Welding, FSpW) é um processo de união no estado sólido semelhante ao FSSW. A grande evolução do FSpW consiste no preenchimento completo da junta soldada, não deixando ao final do processo um furo na superfície da chapa, que atua inevitavelmente como um concentrador de tensão e de corrosão. Desenvolvido pelo centro de pesquisa GKSS Forschungszentrum (atual HelmholtzZentrum Geesthacht) na Alemanha [18], o processo é realizado em quatro etapas distintas por uma ferramenta cilíndrica nãoconsumível constituída por três componentes com sistemas de atuação independentes. O componente de maior diâmetro recebe o nome de anel de fixação, atuando na manutenção do contato entre as chapas a serem soldadas e na prevenção da perda de material (rebarba), e envolve a camisa e o pino, os quais rotacionam e penetram no material de forma independente.

O processo permite duas variantes, seja "penetração da camisa" ou "penetração do pino", dependendo de qual componente da ferramenta é introduzido no material [19]. A Figura 5 apresenta um esquema das quatro etapas da variante "penetração da camisa". Embora a "penetração do pino" seja executada com maior facilidade por demandar intensidades menores de força e torque, o que confere maior vida útil à ferramenta, a "penetração da camisa" fornece uma junta soldada 


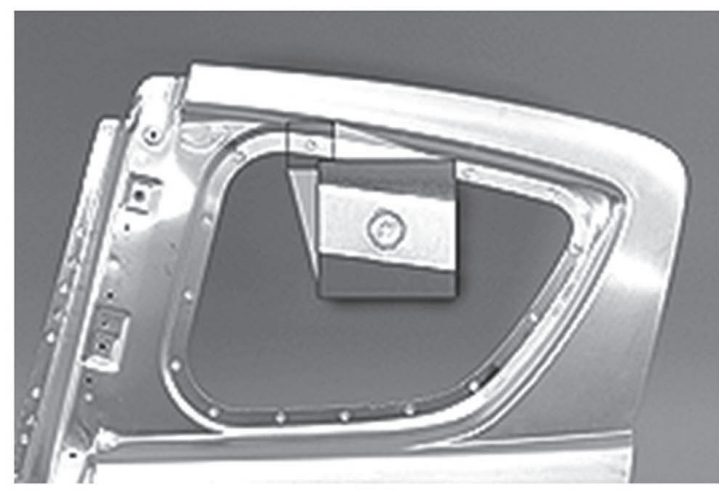

(a)

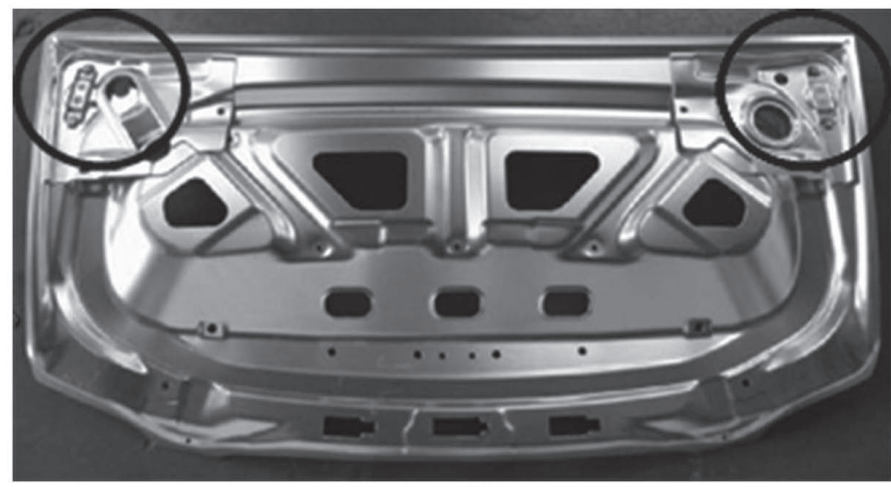

(b)

Figura 4 - Aplicação do FSSW (a) na porta traseira do RX-8 [15] e (b) na tampa do porta-malas do MX-5 [17].

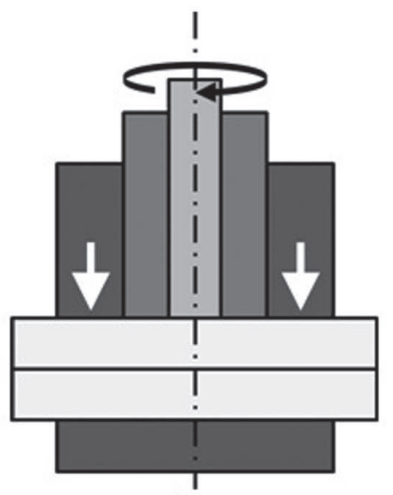

(a)

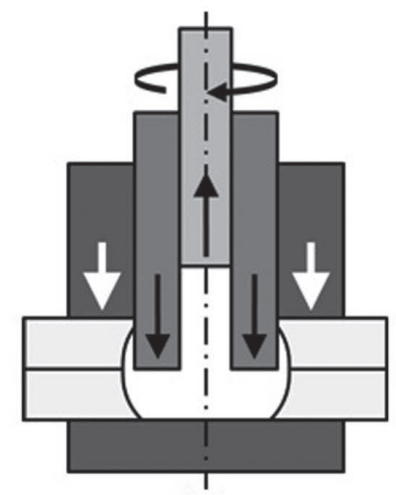

(b)

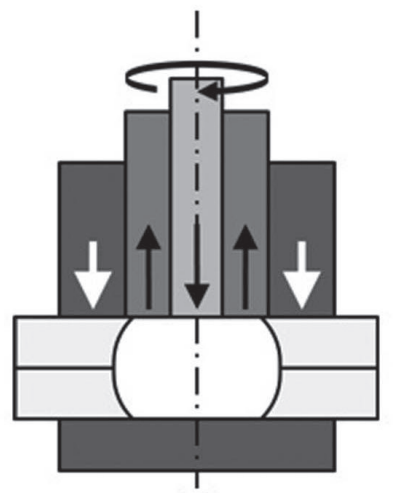

(c)

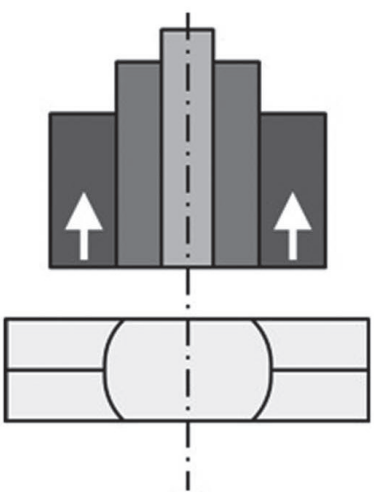

(d)

Figura 5 - Ilustração esquemática da variante "penetração da camisa” do FSpW: (a) fixação e rotação, (b) penetração da camisa e retração do pino, (c) retorno para a superfície e (d) remoção da ferramenta.

de tamanho maior e, consequentemente, de resistência mecânica mais elevada [20].

Enquanto as chapas sobrepostas são pressionadas pelo anel de fixação, o pino e a camisa rotacionam na mesma direção até atingir a velocidade desejada. A aplicação de uma força axial promove a penetração da camisa até uma profundidade pré-determinada, ao mesmo tempo em que o pino é retraído. $\mathrm{O}$ material plastificado pelo calor decorrente do atrito entre o componente penetrante da ferramenta e a superfície do material escoa para a cavidade criada pela retração do pino. Um tempo de operação dos componentes em rotação nas respectivas posições também é utilizado com o intuito de otimizar a mistura do material. O retorno do pino e da camisa para a superfície da chapa empurra o material anteriormente deslocado para o nível superficial, obtendo-se uma região de solda completamente preenchida com perda de material praticamente nula. As etapas da variante "penetração do pino" são semelhantes, exceto pela cavidade na ferramenta ser resultante da penetração do pino e retração da camisa.

O movimento do material plastificado em diversos ensaios durante a variante "penetração do pino" é apresentado na Figura 6, na qual as linhas pretas representam uma folha de Al inicialmente disposta entre as chapas [21]. Durante a penetração completa e a meia penetração do pino, o fluxo ocorre verticalmente para cima conforme a respectiva penetração se processa. No caso da penetração completa do pino com posterior meia retração, a redução na largura do furo indica o movimento do material de volta para a cavidade deixada pelo pino. No processo completo, com ou sem tempo de operação, o material forçado pela camisa promove o preenchimento total da junta. O fluxo se assemelha a um processo de extrusão inversa, havendo também um movimento de mistura significativo do material localizado logo abaixo e em contato ou próximo à superfície cilíndrica do pino. A extensão total da ZM na direção radial é relativamente pequena e corresponde à microestrutura constituída pelo refinamento dos grãos [22,23].

Segundo os padrões microestruturais dos processos no estado sólido derivados do FSW, uma junta típica obtida por FSpW apresenta ZM, ZTMA e ZTA [24,25], conforme mostrado na Figura 7. Novamente as delimitações entre as diferentes zonas de solda é uma aproximação. De maneira semelhante às características apresentadas anteriormente para o FSSW, a ZM é composta por pequenos grãos recristalizados e equiaxiais como consequência da recristalização dinâmica, a ZTMA possui uma estrutura altamente deformada em um padrão de fluxo ascendente e a ZTA não difere substancialmente do MB exceto por alguma transformação resultante do aumento da temperatura. Nota-se claramente o preenchimento praticamente completo da junta soldada, o que resulta em uma extensa área de solda efetiva.

\section{Desempenho Mecânico}

O desempenho das juntas soldadas por FSSW e FSpW é assunto de diversas investigações em razão do enorme potencial de aplicação das tecnologias na união de ligas leves pelos setores automobilístico e aeronáutico. As características mecânicas 


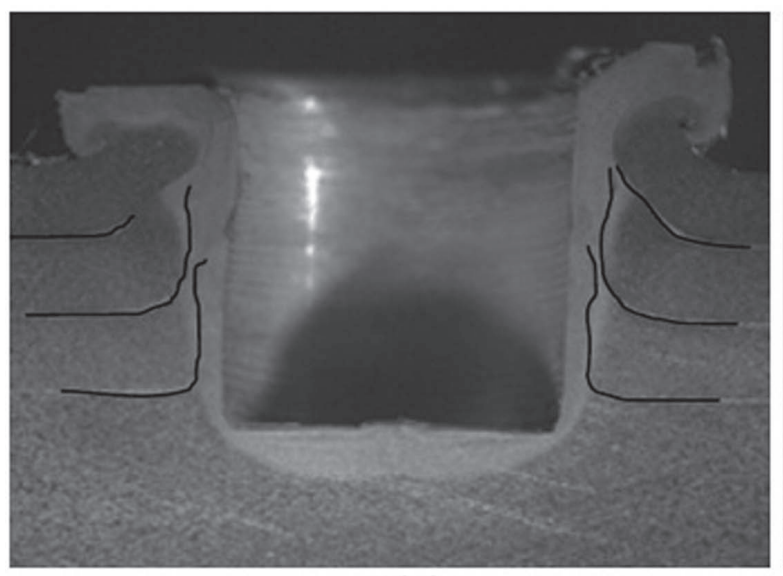

(a)

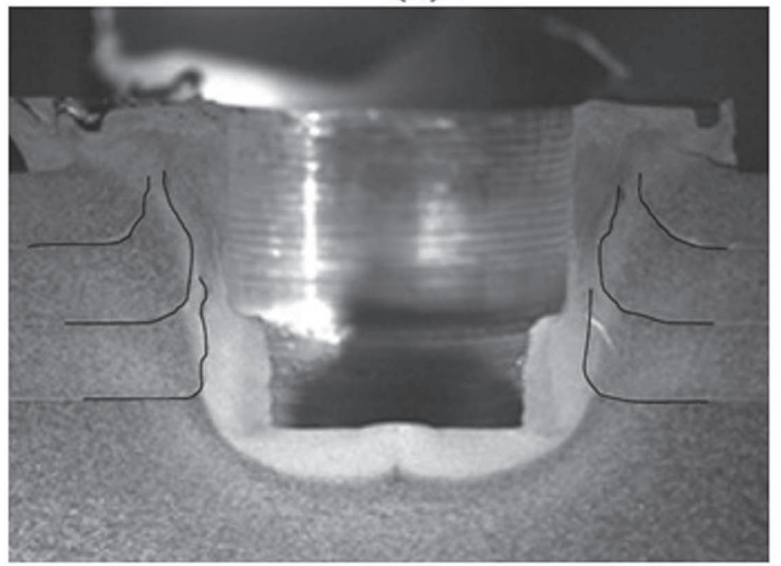

(c)

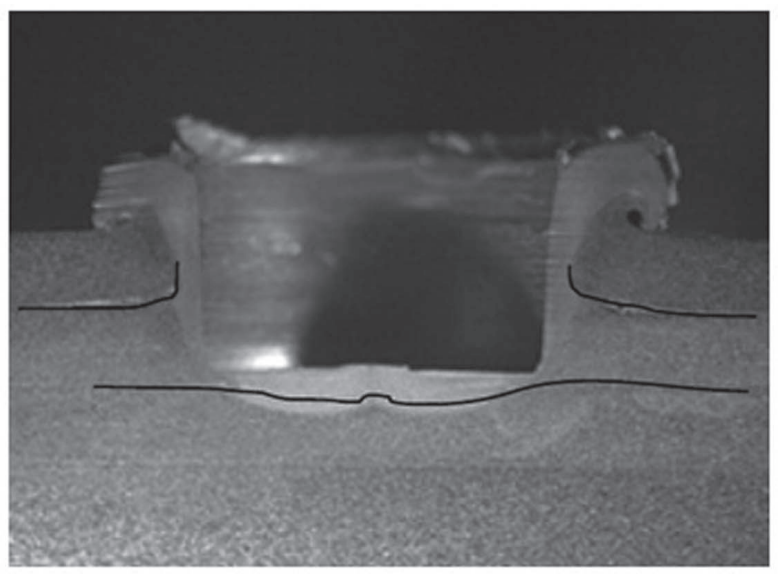

(b)

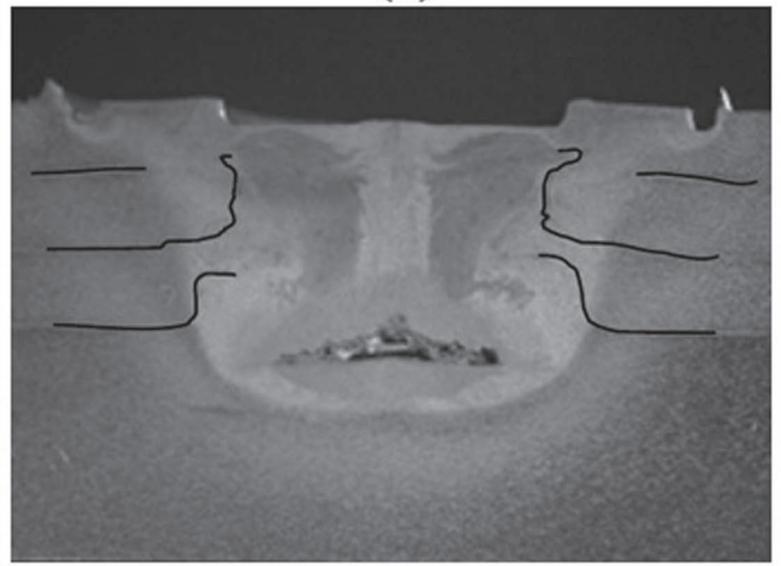

(d)

Figura 6 - Fluxo de material na variante "penetração do pino" a 1800 rpm: (a) penetração completa, (b) meia penetração, (c) penetração completa e meia retração e (d) processo completo [21].

de uma junta sobreposta por ponto são usualmente avaliadas mediante a solicitação da solda em cisalhamento. A Figura 8 apresenta alguns dos melhores valores de tensão de cisalhamento disponíveis na literatura para ligas de $\mathrm{Al} \mathrm{e} \mathrm{Mg.} \mathrm{Embora} \mathrm{não} \mathrm{exista}$ uma metodologia padrão para a obtenção da tensão máxima de uma solda por ponto a partir da respectiva carga, a tensão de cisalhamento é aqui definida como aproximadamente a razão entre a carga ou força de cisalhamento e a área nominal efetiva da junta (correspondente ao diâmetro externo do componente da ferramenta). Este procedimento é adotado com frequência nos casos em que a área verdadeira da junta soldada não é possível de ser medida.

É evidente que o preenchimento completo da região de solda
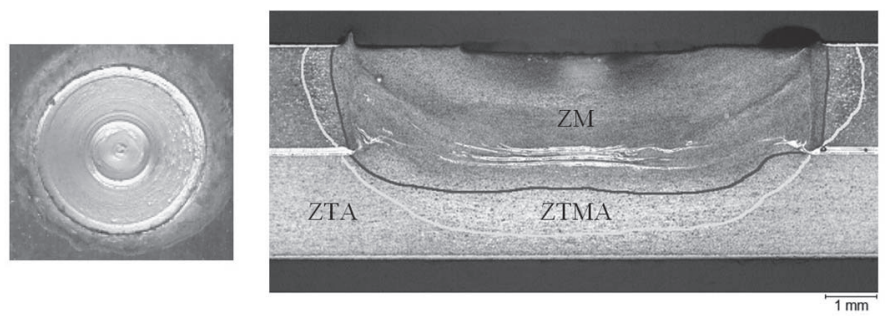

Figura 7 - Aspecto visual e macrografia da seção transversal de uma junta de Al 2024-T4 soldada por FSpW [24]. promove uma melhoria significativa no desempenho mecânico da junta como resultado da obtenção de uma maior área nominal efetiva. O furo deixado na superfície do material após o processo FSSW pode atuar como uma região de concentração de tensão, reduzindo a qualidade da solda. Os valores da tensão de cisalhamento das juntas obtidas por FSpW são superiores tanto para o Al quanto para o Mg. No caso da liga de Mg AZ31 laminada, por exemplo, a tensão de cisalhamento passa de aproximadamente $41 \mathrm{MPa}$ em uma junta soldada por FSSW

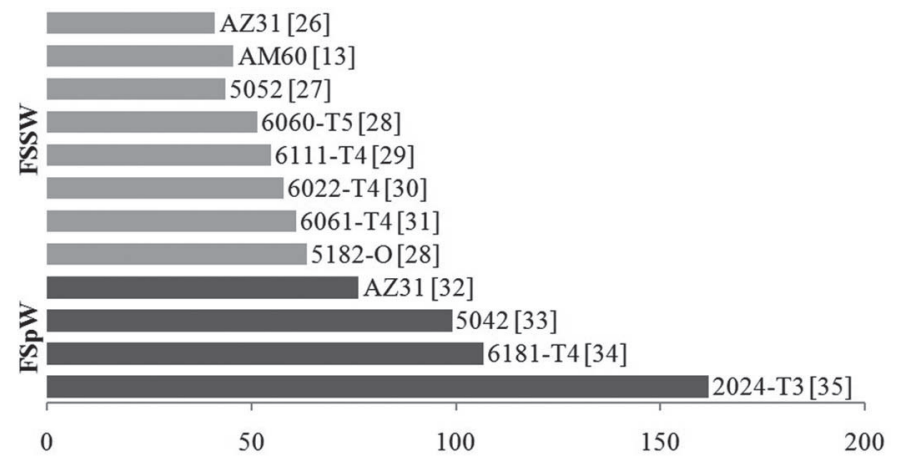

Figura 8 - Dados de tensão de cisalhamento de diversas juntas de $\mathrm{Al}$ e $\mathrm{Mg}$ (Refs. nas barras). 
para aproximadamente $76 \mathrm{MPa}$ em uma soldada por FSpW, o que representa um acréscimo na resistência ao cisalhamento de cerca de $85 \%$. O grande destaque é dado para a liga de Al 2024-T3, amplamente empregada nas fuselagens e em outras estruturas de aeronaves. Embora apresente uma soldabilidade por fusão bastante precária, tendo sido inclusive considerada não-soldável, esta liga mostra uma boa compatibilidade com o processo FSpW, fornecendo juntas com um desempenho sob cisalhamento altamente satisfatório.

Sendo um processo de soldagem por ponto, o FSpW torna-se naturalmente um substituto potencial para o RSW e a rebitagem, que são técnicas consolidadas na indústria automobilística e aeronáutica, respectivamente. Dessa forma, é válida uma comparação entre o maior valor de tensão de cisalhamento da Figura 8, que ocorre para a liga de Al 2024T3, e os requisitos mínimos de resistência estabelecidos pelas normas SAE MAS-W-6858 (referente ao RSW) [36] e MILHDBK-5H (referente à rebitagem) [37]. A Figura 9 apresenta as especificações mínimas de resistência para as tecnologias de união em questão. Os dados da curva do processo RSW referemse às ligas de $\mathrm{Al}$ cujos valores da tensão limite de resistência à tração são superiores a $386 \mathrm{MPa}$, que é o caso da liga 2024$\mathrm{T} 3$, com um valor de aproximadamente $425 \mathrm{MPa}$ [35]. Quanto à curva de rebitagem, os dados referem-se a rebites do tipo sólido do mesmo material.

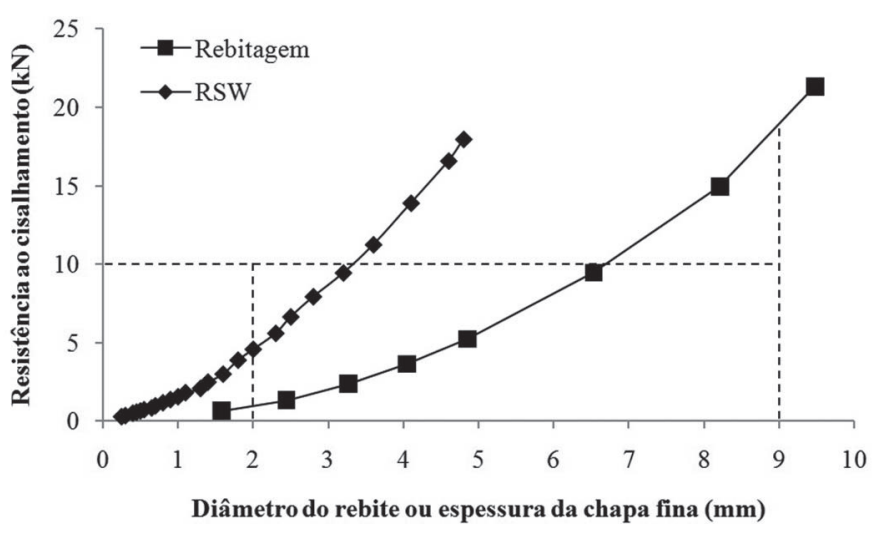

Figura 9 - Carga sob cisalhamento obtida por diferentes técnicas de união para a liga de Al 2024-T3.

Para efeito de comparação, a carga de cisalhamento da liga de Al 2024-T3 da Figura 8 equivale a aproximadamente $10,1 \mathrm{kN}$ em chapas de $2 \mathrm{~mm}$ de espessura [35]. Considerando uma junta sobreposta obtida por RSW em chapas de mesma dimensão, a resistência ao cisalhamento estabelecida é de $5 \mathrm{kN}$. Uma vez que a resistência fornecida é duas vezes superior, o processo FSpW mostra, neste caso, a possibilidade de substituição direta do RSW. Considerando agora um rebite de Al 2024-T3 com 9 mm de diâmetro, que equivale ao diâmetro da camisa da ferramenta [35], a resistência especificada é de aproximadamente $19 \mathrm{kN}$. Portanto, o FSpW ainda requer uma melhor otimização de processo para assegurar uma aplicação confiável no setor aeronáutico em substituição à técnica de rebitagem.

\section{Conclusões}

As principais características dos processos de soldagem por ponto no estado sólido derivados da soldagem por fricção e mistura (FSW) foram apresentadas, bem como algumas aplicações e potenciais aplicações que envolvem ligas leves de alumínio e magnésio. No processo de soldagem por fricção e mistura por ponto (FSSW), a ferramenta em rotação é introduzida no material e posteriormente retraída, formando uma região microestrutural recristalizada dinamicamente que promove a conexão entre as placas em configuração de sobreposição. Embora a quantidade de defeitos metalúrgicos na junta soldada seja reduzida, a ferramenta deixa um furo remanescente no centro da junta soldada em função da perda de material na forma de rebarba. De modo similar, o processo de soldagem por fricção por ponto (FSpW) promove a conexão entre as placas sobrepostas mediante a formação de uma zona recristalizada dinamicamente. Neste caso, a ferramenta possui componentes que atuam de maneira independente, havendo a formação de uma cavidade pela retração de uma das partes onde se aloca o material deslocado pela penetração da outra parte. A perda de material é insignificante, sendo o preenchimento completo da junta após o ciclo de soldagem a grande vantagem em relação ao FSSW.

Além de serem compatíveis com os requisitos ambientais, os processos de soldagem por ponto no estado sólido possuem uma grande facilidade de automação, não exigindo altos investimentos em infra-estrutura, e têm elevada eficiência energética, pois consomem energia basicamente para a movimentação da ferramenta. Estas técnicas possuem um enorme potencial de aplicação nas indústrias automobilística e aeronáutica, fazendo frente às tecnologias RSW e rebitagem, respectivamente. O FSSW já encontra algumas aplicações na indústria automobilística. A análise de alguns dados de desempenho das juntas sob cisalhamento mostrou a superioridade do processo FSpW em relação tanto ao FSSW quanto ao RSW, podendo substituí-los diretamente na união de ligas metálicas leves. Embora contribuam com a redução do peso das estruturas, os processos ainda estão aquém das exigências de desempenho do setor aeronáutico, necessitando ainda de melhores otimizações para a possibilidade de substituição dos rebites. No entanto, a aplicação em situações que não exigem grandes valores de resistências pode ser viável.

\section{Agradecimentos}

Os autores agradecem à Fundação de Amparo à Pesquisa do Estado de São Paulo (FAPESP) e ao Conselho Nacional de Desenvolvimento Científico e Tecnológico (CNPq) pelo apoio financeiro.

\section{Referências}

[1] AGHION, E.; BRONFINA, B.; ELIEZERB, D. The role of the magnesium industry in protecting the environment. Journal of Materials Processing Technology, v. 117, n. 3, p. 381-385, 2001. [2] THOMAS, W.M. et al. Friction Stir Butt Welding. GB Patent 9125978-8, 6 dez. 1991. 
[3] MISHRA, R.S.; MA, Z.Y. Friction stir welding and processing. Materials Science and Engineering R, v. 50, n. 1-2, p. 1-78, 2005.

[4] THREADGILL, P.L. Friction stir welding - the state of the art. Relatório Técnico TWI, n. 7417.01/99/1012.03, 1999.

[5] LEE, W.B.; YEON, Y.M.; JUNG, S.B. Joint properties of friction stir welded AZ31B-H24 magnesium alloy. Materials Science and Technology, v. 19, n. 6, p. 785-790, 2003.

[6] PAN, T.-Y.; ZHU, W.; SCHWARTZ, W.J. Spot Friction Welding - A New Joining Method for Aluminum Sheets. In: International Automotive Body Congress (IABC), 2005, Ann Arbor, EUA. Proceedings... 2005. v. 2, p. 95-99.

[7] IWASHITA, T. Method and Apparatus for Joining. US Patent 6601751-B2, 5 ago. 2003.

[8] HORIE, S. et al. Experimental investigation of material flow during friction stir spot welding. Science and Technology of Welding and Joining, v. 15, n. 8, p. 666-670, 2010.

[9] YANG, Q. et al. Material flow during friction stir spot welding. Materials Science and Engineering A, v. 527, n. 16-17, p. 4389-4398, 2010.

[10] SU, P. et al. Material flow during friction stir spot welding. Science and Technology of Welding and Joining, v. 11, n. 1, p. 61-71, 2006.

[11] SU, P. et al. Intermixing in Dissimilar Friction Stir Spot Welds. Metallurgical and Materials Transactions A, v. 38, n. 3, p. 584-595, 2007.

[12] LIN, P.-C; LIN, S.-H.; PAN, J. Microstructures and Failure Mechanisms of Spot Friction Welds in Lap-Shear Specimens of Aluminum 6111-T4 Sheets. SAE International, 2004-01-1330, 2004. [13] SU, P.; GERLICH, A.; NORTH, T.H. Friction Stir Spot Welding of Aluminum and Magnesium Alloy Sheets. SAE International, 2005-01-1255, 2005.

[14] GERLICH, A.; AVRAMOVIC-CINGARA, G.; NORTH, T.H. Stir Zone Microstructure and Strain Rate during Al 7075T6 Friction Stir Spot Welding. Metallurgical and Materials Transactions A, v. 37, n. 9, p. 2773-2786, 2006.

[15] HANCOCK, R. Friction Welding of Aluminum Cuts Energy Costs by 99\%. Welding Journal, v. 83, n. 2, p. 40, 2004. [16] Mazda Develops World's First Aluminum Joining Technology Using Friction Heat. Mazda Media Release, 27 fev. 2003. Disponível em: <http://www.mazda.com/publicity/ release/2003/200302/0227e.html>. Acesso em: 20 jul. 2011.

[17] Mazda Develops World's First Steel and Aluminum Joining Technology Using Friction Heat. Mazda Media Release, 02 jun. 2005. Disponível em: <http://www.mazda.com/publicity/ release/2005/200506/050602. html>. Acesso em: 20 jul. 2011.

[18] SCHILlinG, C.; DOS SANTOS, J. Method and Device for Linking at Least Two Adjoining Work Pieces by Friction Welding. International Patent Publication WO/2001/036144, 25 mai. 2005. [19] DA SILVA, A.A.M. et al. Preliminary Investigation on Microstructural Features and Mechanical Performance of FSpW of Al Alloys. In: IIW International Seminar on Friction based Spot Welding Processes, 2007, Geesthacht, Alemanha. Proceedings... 2007.

[20] ARBEGAST, W.J. Refill Friction Stir Spot Welding of Aluminium Alloys. In: IIW International Seminar on Friction based Spot Welding Processes, 2007, Geesthacht, Alemanha. Proceedings... 2007.
[21] MUCI-KÜCHLER, K.H. et al. Numerical Simulation of the Friction Stir Spot Welding Process. SAE International, 200501-1260, 2005.

[22] MUCI-KÜCHLER, K.H. et al. Visualization of Material Flow in Friction Stir Spot Welding. SAE International, 2005-013323, 2005.

[23] ITAPU, S.K.; MUCI-KÜCHLER, K.H. Visualization of Material Flow in the Refill Friction Stir Spot Welding Process. SAE International, 2006-01-1206, 2006.

[24] ROSENDO, T.S. et al. Preliminary Investigation on Friction Spot Welding of Alclad 2024-T4 Aluminum Alloy. In: XXXIII Congresso Nacional de Soldagem (CONSOLDA), 2007, Caxias do Sul. Anais... 2007.

[25] DA SILVA, A.A.M. et al. Performance Evaluation of 2-mm thick alclad AA2024 T3 Aluminium Alloy Friction Spot Welding. SAE International, 2007-01-3812, 2007.

[26] YANG, Q. et al. Microstructure and Mechanical Properties of Friction Stir Spot Welded AZ31 Mg Alloy. In: $7^{\text {th }}$ International Friction Stir Welding Symposium, 2008, Awaji Island, Japão. Proceedings... 2008.

[27] LEE, C.-Y. et al. Joint Characteristics of Spot Friction Stir Welded $5052 \mathrm{Al}$ Alloy Sheet. Advanced Materials Research, v. 15-17, p. 345-350, 2007.

[28] LATHABAI, S. et al. Friction Stir Spot Welding of Automotive Lightweight Alloys. In: $7^{\text {th }}$ International Conference on Trends in Welding Research, 2005, Pine Mountain, EUA. Proceedings... 2005.

[29] ARUL, S.G. et al. Experimental study of joint performance in spot friction welding of 6111-T4 aluminium alloy. Science and Technology of Welding and Joining, v. 13, n. 7, p. 629-637, 2008. [30] OKAMOTO, K.; HUNT, F. Development of Friction Stir Welding Technique and Machine for Aluminum Sheet Metal Assembly. SAE International, 2005-01-1254, 2005.

[31] TOZAKI, Y.; UEMATSU, Y,; TOKAJI, K. Effect of tool geometry on microstructure and static strength in friction stir spot welded aluminium alloys. International Journal of Machine Tools and Manufacture, v. 47, n. 15, p. 2230-2236, 2007.

[32] CAMPANELLI, L.C. et al. Preliminary Investigation on Friction Spot Welding of AZ31 Magnesium Alloy. In: $7^{\text {th }}$ International Conference on Advanced Materials (THERMEC), 2011, Quebec, Canadá.

[33] TIER, M. et al. The influence of weld microstructure on mechanical properties of refill friction spot welding of 5042 aluminium alloy. In: $7^{\text {th }}$ International Friction Stir Welding Symposium, 2008, Awaji Island, Japão. Proceedings... 2008.

[34] ROSENDO, T. et al. Mechanical and microstructural investigation of friction spot welded AA6181-T4 aluminium alloy. Materials and Design, v. 32, n. 3, p. 1094-1100, 2011.

[35] AMANCIO-FILHO, S.T. et al. Preliminary Investigation of the Microstructure and Mechanical Behaviour of 2024 Aluminium Alloy Friction Spot Welds. Materials Transactions, v. 52, n. 5, p. 985-991, 2011.

[36] SOCIETY OF AUTOMOTIVE ENGINEERING. AMSW-6858A: Welding, Resistance: Spot and Seam. Warrendale, EUA, 2000.

[37] U.S. DEPARTMENT OF DEFENSE. Metallic Materials and Elements for Aerospace Vehicle Structures. Military Handbook, MIL-HDBK-5H, 1998. cap. 8. 